\title{
PERFORMANCE REACH OF THE "PHASE 1" LHC COLLIMATION SYSTEM
}

\author{
G. Robert-Demolaize, R. Assmann, C. Bracco, S. Redaelli, T.Weiler \\ CERN, Geneva, Switzerland
}

\begin{abstract}
State-of-the-art tracking tools have been developed for detailed LHC collimation and beam loss studies. This includes full chromatic treatment of both beam lines and error models. This paper reviews the main results on the performance reach of the multi-stage LHC collimation system that is being installed in the LHC. Limitations on the allowed proton loss rates and the stored intensity can be derived from the comparison of local losses with estimated quench limits for the superconducting magnets. The origins of the cleaning-related performance limitations are presented and possible improvements are discussed.
\end{abstract}

CERN, 


\title{
PERFORMANCE REACH OF THE "PHASE 1" LHC COLLIMATION SYSTEM
}

\author{
G. Robert-Demolaize, R. Assmann, C. Bracco, S. Redaelli, T. Weiler, CERN, Geneva, Switzerland
}

\section{Abstract}

State-of-the-art tracking tools have been developed for detailed LHC collimation and beam loss studies. This includes full chromatic treatment of both beam lines and error models. This paper reviews the main results on the performance reach of the multi-stage LHC collimation system that is being installed in the LHC. Limitations on the allowed proton loss rates and the stored intensity can be derived from the comparison of local losses with estimated quench limits for the superconducting magnets. The origins of the cleaning-related performance limitations are presented and possible improvements are discussed.

\section{INTRODUCTION}

The LHC collimators are located in two warm insertions dedicated to beam cleaning and are used to intercept beam halo [1]. A small fraction of the halo leaks out and gets lost at characteristic locations around the ring. The performance of the system is described by its local cleaning inefficiency $\eta$ :

$$
\eta=\frac{\text { number of protons lost in the machine aperture }}{\text { number of protons absorbed by the system } \times \Delta \mathrm{L}},
$$

with $\Delta L$ a given length over which losses are distributed, which will be $10 \mathrm{~cm}$ in the following. Critical loss locations are spotted by comparing the local inefficiency values with the magnet quench levels for estimated minimal beam lifetimes $[2,3]$. The nominal reference cases are defined with the parameters listed in Tables 1 and 2.

Table 1: Optics parameters of the simulated nominal cases.

\begin{tabular}{|c|c|c|c|}
\hline Case & E [TeV] & IR 1 \& 5 & IR 2 \& 8 \\
\hline Injection & 0.45 & $\beta^{*}=17 \mathrm{~m}$ & $\beta^{*}=10 \mathrm{~m}$ \\
\hline Collision & 7 & $\beta^{*}=0.55 \mathrm{~m}$ & $\beta^{*}=10 \mathrm{~m}$ \\
\hline
\end{tabular}

Table 2: Minimal beam lifetimes $\tau$ and corresponding required local inefficiencies for the simulated nominal cases at the quench limit.

\begin{tabular}{|c|c|c|}
\hline Case & $\tau[\mathbf{h}]$ & $\tilde{\eta}_{\text {quench }}\left[\mathbf{m}^{-1}\right]$ \\
\hline Injection & 0.1 & $10^{-3}$ \\
\hline Collision & 0.2 & $2 \times 10^{-5}$ \\
\hline
\end{tabular}

A modified version of the well-established SIXTRACK code was developed to perform tracking studies that would include scattering routines for beam collimation [4]. In addition, a detailed aperture model was set up to check for 01 Circular Colliders proton losses along the machine in steps of $\Delta L$ [5]. These two programs are then used to check for longitudinal loss locations over the machine when tracking more than 5 millions of particles over 200 turns.

\section{TRACKING RESULTS}

\section{Simulations in the ideal machine case}

In its early stages, the LHC collimation system was designed as a two-stage one, i.e. with only primary and secondary collimators. Two LHC warm insertions are dedicated to cleaning: IR3 for momentum cleaning, IR7 for betatron cleaning. Studies of the efficiency of the betatron cleaning system highlighted the need for additional collimators to provide dedicated protection to specific regions of the machine [6], namely:

- active absorbers, for the dispersion suppressor region downstream of the IR7 collimators,

- target collimators, for the injection (IR2, IR8) and beam dump (IR6) regions,

- tertiary collimators, for the triplet magnets in the four experimental insertions (IR1, IR2, IR5, IR8).

The full Phase 1 LHC collimation system includes all collimators from the initial system plus the new ones described above. Results from tracking show that the collision energy case $(7 \mathrm{TeV})$ is the most critical one. Major improvements are achieved with the full Phase 1 system, as seen in Figure 1. The peaks indicating halo losses (red/blue peaks for warm/cold regions) in the initial system case are replaced by the absorption rates at the additional collimators (green peaks). One can notice that the IR7 dispersion suppressor region downstream of the cleaning system still show local losses over the quench threshold, by about a factor 2. This marks a limitation in the level of performance of the LHC collimation system: these losses can never be avoided as they are induced by single-diffractive events experienced by the halo protons impacting on the collimator materials.

\section{Simulations with error models}

In both of the previous cases, the machine settings were taken as ideal, i.e. no orbit or beta-beating errors, all lattice elements perfectly aligned and collimators always centered around the closed orbit. The tracking tools developed for the LHC collimation studies allow implementing error models to simulate realistic machine conditions. For one T19 Collimation and Targetry 

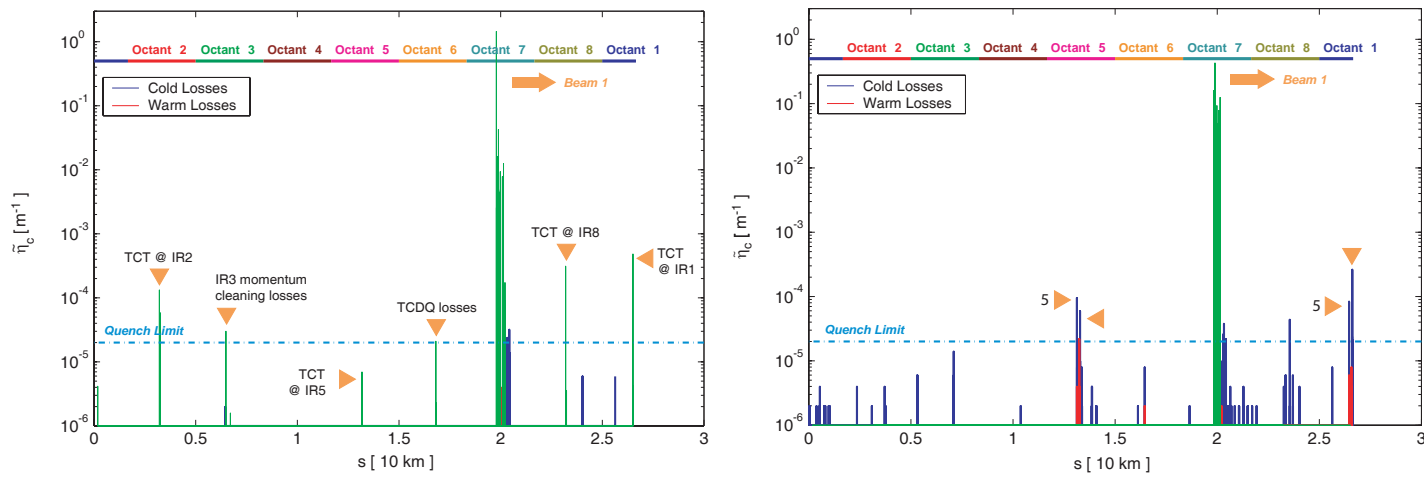

Figure 1: Beam loss map at collision energy for the initial (left) and full Phase 1 (right) LHC collimation system. The simulated halo is horizontal. Results are shown for Beam 1 only.

of the scenarios, nominal closed orbit perturbations are applied to the model of the machine. The peak amplitudes of the error are derived from experiences from LEP operations [7] and are reported in Table 3 for each beam energy.

Table 3: Closed orbit tolerances for the nominal optics.

\begin{tabular}{|c|c|c|}
\hline Case & Arc tolerances & IR tolerances \\
\hline Injection & $\pm 4 \mathrm{~mm}$ & $\pm 4 \mathrm{~mm}$ \\
\hline Collision & $\pm 4 \mathrm{~mm}$ & $\pm 3 \mathrm{~mm}$ \\
\hline
\end{tabular}
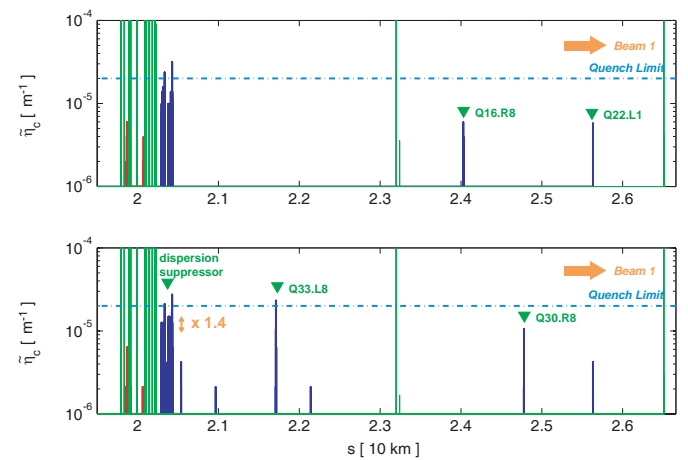

Figure 2: Comparison of beam losses for a horizontal halo at collision energy downstream of IR7 between the ideal closed orbit (top) and a horizontal orbit error (bottom). Results are shown for Beam 1 only.

Simulations performed using this error model show that the level of local proton losses around the machine are all below the quench threshold in the injection energy case. At $7 \mathrm{TeV}$, the dispersion suppressor downstream of the collimators in IR7 is still the most critical region. Figure 2 even shows a $40 \%$ increase in terms of local cleaning inefficiency at the Q11 magnet.

\section{EXPECTED PERFORMANCE REACH WITH THE PHASE 1 SYSTEM}

\section{Cleaning efficiency of the collimation system}

To determine how effective the cleaning system is, two relevant parameters can be checked: the maximum inten01 Circular Colliders sity that can be stored in the machine, and the maximum allowed proton loss rates. These values can be derived by comparing the local cleaning efficiency values around the machine (as displayed in the longitudinal loss maps) with the simplified quench levels for each energy (listed in Table 2 .

Table 4 lists the main results from the tracking studies for all considered scenario and both beam lines. At injection energy $(450 \mathrm{GeV})$, the machine can be operated safely with the nominal LHC beam intensity (2808 bunches with $1.15 \times 10^{11}$ protons per). A reduction of more than a factor 2 in maximum allowed intensity can be noted though when nominal orbit conditions are taken into account. In the collision energy case $(7 \mathrm{TeV})$, studies of the ideal machine scenario show a limitation of $43 \%$ of the nominal beam intensity, while this value drops to $27 \%$ when orbit perturbations are included in the simulations.

\section{Analysis of the scattering data}

Some regions of the machine require extra attention due to specific conditions, e.g. beam dump region and the triplet quadrupoles in the experimental insertions. The tracking tools developed for collimation studies also provide output files with the $6 \mathrm{D}$ coordinates of the particles suffering inelastic interactions in the collimator materials. This allow studying the particle showers induced by these scattering processes and check the amount of energy deposited in downstream elements.

Figure 3 shows an example of a transverse map of deposited energy in the coils of one of the quadrupoles downstream of the beam dump collimators, as obtained from FLUKA simulations. Two values have to be considered regarding local quench protection: the local peak energy density and the total power deposition. It can be seen that both values are safe in the example shown, but have to be taken into account along with the statistics from direct proton losses (derived from the longitudinal loss maps) when looking for critical loss locations.

Background levels in detectors could also increase significantly due to particle showers coming from the tertiary

T19 Collimation and Targetry 
Table 4: Summary on the performance level for different machine scenarios using the full Phase 1 LHC collimation system. The performance is estimated in both maximum allowed intensity and maximum allowed loss rate $R_{\text {loss }}^{\max }$.

\begin{tabular}{|c|c|c|c|c|c|}
\hline Scenario & $\begin{array}{c}\text { Energy } \\
{[\mathrm{TeV}]}\end{array}$ & $\begin{array}{c}\tilde{\eta}_{\mathbf{c}, \max }^{\text {cold }} \\
{\left[10^{-5} \mathrm{~m}^{-1}\right]}\end{array}$ & $\begin{array}{c}\tau \\
{[\mathrm{h}]}\end{array}$ & $\mathrm{I}_{\max } / \mathrm{I}_{\text {nom }}$ & $\begin{array}{c}\mathbf{R}_{\text {loss }}^{\max } \\
{\left[10^{10} \text { protons/s }\right]}\end{array}$ \\
\hline Ideal machine & 0.45 & $18.65 \pm 1.96$ & 0.1 & $5.38 \pm 0.57$ & $376.34 \pm 39.72$ \\
\cline { 2 - 6 } & 7.00 & $4.60 \pm 0.96$ & 0.2 & $0.43 \pm 0.09$ & $16.52 \pm 3.44$ \\
\hline $\begin{array}{c}\text { Ideal machine } \\
\text { with nominal orbit }\end{array}$ & 0.45 & $40.60 \pm 2.95$ & 0.1 & $2.46 \pm 0.18$ & $172.41 \pm 12.51$ \\
\cline { 2 - 6 } & 7.00 & $7.45 \pm 1.21$ & 0.2 & $0.27 \pm 0.04$ & $10.20 \pm 1.66$ \\
\hline
\end{tabular}

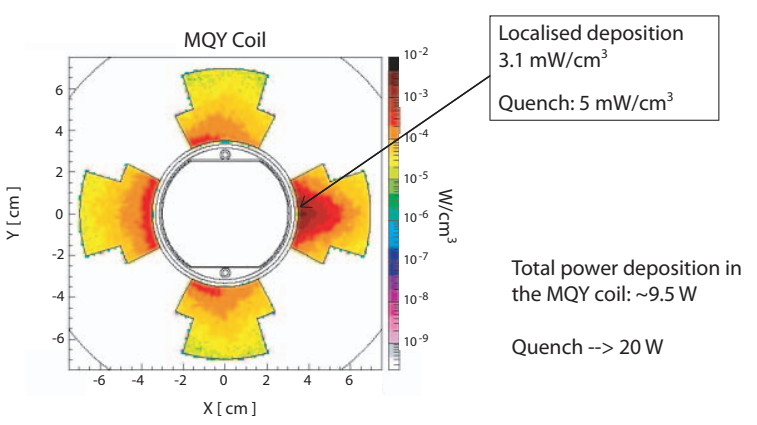

Figure 3: Transverse map of energy deposition in the MQY magnet downstream of the beam dump collimators. This plot gives the peak deposited energy and compares it with the estimated quench threshold for the considered element. Courtesy of L. Sarchiapone.

collimators used to protect the triplet quadrupoles. Studies were performed for IR8 [8], which is the closest experimental region to the IR7 cleaning insertion when following the Beam 1 direction. Preliminary results are shown in Figure 4: it appears that additional shielding walls are required close to the detector in order to reduce the muon and hadron levels induced by the tertiary collimators.
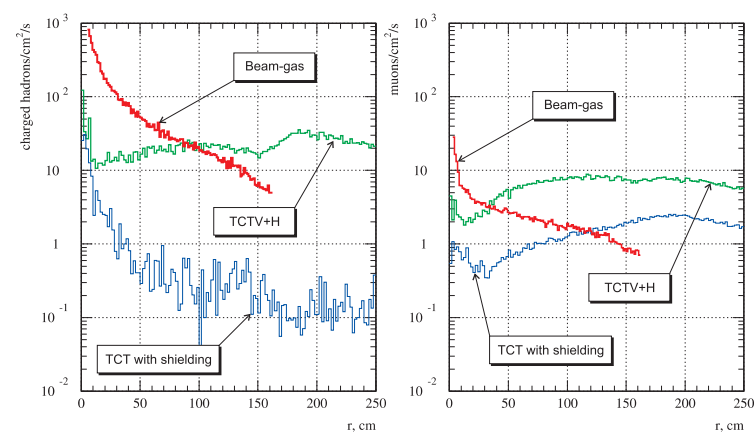

Figure 4: Radial distribution of the flux density of charged hadrons (left) and muons (right) issued from the tertiary collimators in the IR8 region. Beam-gas data is given as comparison. Courtesy of G. Corti and V. Talanov.

\section{CONCLUSION}

Intensive tracking studies were performed to get an estimate of the performance of the Phase 1 LHC collimation system. Under nominal orbit conditions at top energy, the 01 Circular Colliders
LHC may still be operated safely at the quench threshold if there is a maximum of $27 \%$ of the nominal beam intensity stored in the machine. On top of that, one should also include the results from studies on energy deposition and background levels, which may change the limitations on local cleaning inefficiencies.

Additional error models can also be applied to the machine in further studies, e.g. nominal $\beta$-beating, coupling, or offsets in the mechanical settings of collimators. Tighter limitations should be expected after all detailed models are included. To comply with these probable new restrictions, a Phase 2 design of the system is being studied. The major changes from Phase 1 are a different material (using copper instead of a carbon composite to improve the absorption rate) and a rotative mechanical design for the secondary collimators. These changes should allow running the LHC at his ultimate settings with a $40 \%$ increase in beam intensity compared to the nominal value (up to 2808 bunches of $1.6 \times 10^{11}$ protons).

\section{REFERENCES}

[1] "LHC Design Report", Volume I, Ch. 18, CERN, 2004

[2] R. Assmann: "Collimators and cleaning: could this limit the LHC performance?", Proc. Chamonix 2003.

[3] J.B. Jeanneret, D. Leroy, L. Oberli and T. Trenckler: "Quench levels and transient beam losses in the LHC magnets", CERN-LHC-PROJECT-REPORT-44, 1996.

[4] G. Robert-Demolaize, R. Assmann, S. Redaelli, F. Schmidt: "A new version of SixTrack with collimation and aperture interface", Proc. PAC 2005.

[5] S. Redaelli: "LHC aperture and commissioning of the collimation system", Proc. Chamonix 2005.

[6] G. Robert-Demolaize: "Design and performance optimization of the LHC collimation system", CERN-LHCPROJECT-REPORT-981, see also CERN-THESIS-2006069, 2006.

[7] J. Wenninger: "Quadrupole alignment and closed orbits at LEP: a test ground for LHC", LHC Project Note 104, 1998.

[8] G. Corti, V. Talanov: "Aspects of machine induced background in the LHC experiments", proceedings of the Chamonix XV Workshop, 2006. 